\title{
Ventricular-atrial junction in post hemorrhagic hydrocephalus: a rare case of massive catheter atrial thrombosis
}

\begin{abstract}
Although the infant age is notoriously the hardest period of life to be affected by thromboembolic accidents in the medical literature there are still very few studies on this issue, and therefore the treatment of these pathological processes is, on this stage of life, even today, a real challenge. In fact the role of medical therapy is still debated, either as regards the most appropriate medical procedure to use, or the effectiveness, and because of its limits which were often described and because of there are no protocols which could be really sure or recommended. We describe the case of a patient who came to our observation at the age of 11 months for a severe thrombosis of the ventricle-atrial shunt catheter placed after a post-hemorrhagic hydrocephalus. In our division, the patient was subjected to medical therapy, first with only heparin, then associated with thrombolytics. This case emphasizes the difficulties in the therapeutic management of pediatric patients with thrombosis, it emphasizes the limits of any medical treatment and reiterates the need to guide patients with these phenomena promptly to surgery, which is the only effective and decisive therapy.
\end{abstract}

Keywords: hydrocephalus, thrombosis, infant, thrombolytic, surgery
Volume 8 Issue 2 - 2017

\author{
Spoto S, Alaimo A, Centineo F, Passarello D, \\ Di Spezio D, Fiorentino G, Comparato C \\ Paediatric Cardiology Unit,Arnas Civico Benfratelli Di Cristina, \\ Italy
}

Correspondence: Sabrina Spoto, Paediatric Cardiology Unit, Arnas Civico Benfratelli Di Cristina, Palermo, Italy, Emailsposabry@libero.it

Received: January 22, 2017 | Published: February 03, 2017

\section{Introduction}

The first few days of life of a neonate are the period with the highest incidence of thromboembolic events., ${ }^{1,2}$ Recent studies investigating the epidemiology and the risk factors associated to those events have shown significant differences between newborns,infants and adults. ${ }^{3-5}$ The management of thrombosis in this stage of life is very complicated due to the scarcity of controlled clinical trials on neonates and infants, thus resulting in a lack of information on the suitability of the diagnostic tools, the effectiveness and safety of treatments, and the appropriate observation period needed to check any remote outcomes. ${ }^{3-6,7}$ Moreover, this health issue is expected to become more relevant in the years ahead, due to an increase of the survival rate of newborn, in particular of premature infants and very low birth-weight children, given by the advance in the available medical procedures, such as venous and arterial catheterism.

Therefore, we are presenting a case that emphasizes the complexity of the clinical management in infants with thromboembolism following the guideline suggested by the Group of Neonatal Haematology of the Italian Society of Neonatology and by the Strategic Committee of the Study on Coagulation of the Italian Association of Hematology and Pediatric Oncology. We aim to verify the results obtained just through the suggested pharmacological therapy only, when a neurosurgical intervention is not possible as first approach.

\section{Case report}

Our patient, an extreme preterm twin, was born at the 27th week of gestation with multiple complication. In fact, at birth the newborn was affected by respiratory distress due to surfactant deficiency, anemia, sepsis and posthemorrhagic hydrocephalus. Therefore, at the age of four month he underwent ventriculoperitoneal (VP) shunt. However, after two months from the first surgical intervention, it was necessary to replace the catheter due to infection. Successively, at seven months of life, a malfunction of the VP shunt made necessary proceeding to a surgical removal of the entire VP shunt system and to the positioning of a ventriculoatrial (VA) shunt with OMNISHUNT 80-120 INTEGRA valve. When the infant was 11 month old, hospitalization in our department was necessary because of a fever resisting homebased therapy. We proceeded with an echocardiogram that showed a thrombotic formation on the catheter tip in the right atrium, thus it was decided to begin an anticoagulant therapy with a dose of $1.7 \mathrm{mg} /$ $\mathrm{kg}$ of low-molecular-weight-heparin (LMWH) every 12hours. The ineffectiveness of this treatment was demonstrated by a significant increase of the thrombotic massed,which became jutting over the tricuspid plane and by the onset of an obstacle to the discharge of the superior vena cava (SVC) (max gradient of $25 \mathrm{mmHg}$, average gradient of $18-20 \mathrm{mmHg}$ ) (Figure $1 \& 2$ ).

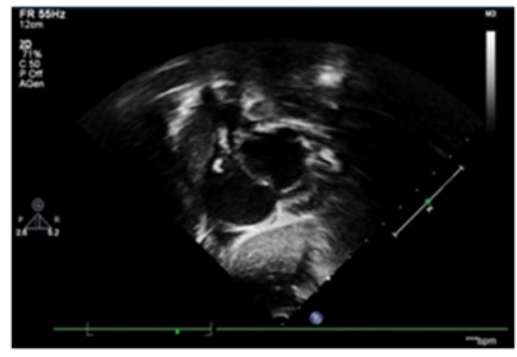

Figure I TTE, subcostal view.

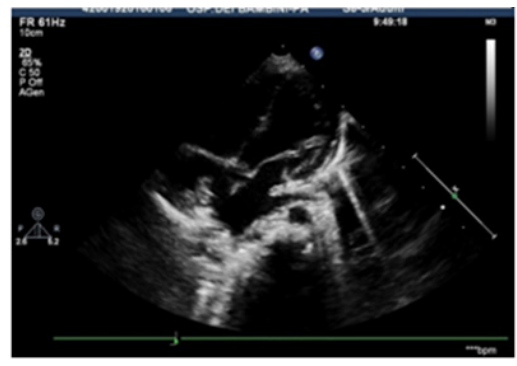

Figure 2 TTE, apical view. 
As a result, we began a treatment with a dose of $7 \mathrm{mg} /$ day $(0.04 \mathrm{mg} / \mathrm{Kg} / \mathrm{h})$ of recombinant tissue plasminogen activator (rTPA), a thrombolytic that acts preferentially on plasminogen bound to fibrin converting it to plasmin. During the administration of this pharmaceutical, it was necessary the infusion of fresh plasma, due to the reduction of fibrinogen values, and the transfusion of concentrated red cell, due to anemia. The therapy with thrombolytic was discontinued after 52 hours given the complete thrombus resolution and the normalization of the SVC flow pattern, and replaced again by LMWH, according to body weight.

Since the persistence of the fever during the treatment, serial blood cultures were performed, resulting positive to Staphylococcus Schleiferi. This lead to a combined treatment of Ampicillin and Rifampicin. However, the suspension of thrombolytic therapy was followed by recurrence and progressive aggravation of thrombosis at the level of the catheter with a new obstruction of the SVC. We therefore decided to transfer immediately the patient to the pediatric neurosurgery unit at the Meyer of Florence for urgent surgical treatment.There, the same anticoagulant treatment was applied and its ineffectiveness was further evidenced by a supervening pulmonary embolism, thus leading to the surgical removal of the VA shunt and the repositioning of VP shunt. It wasn't reported any postsurgical complications (Figure 3)

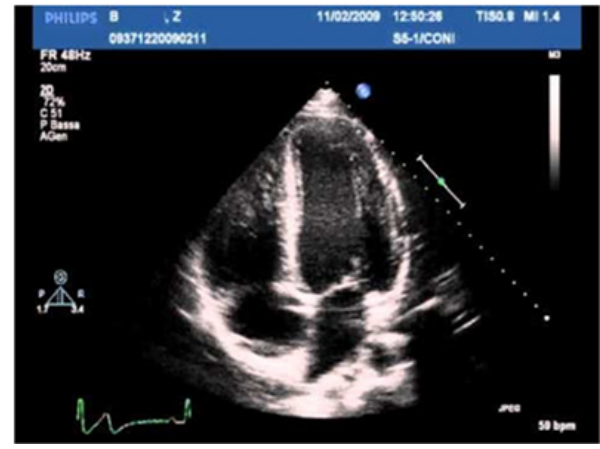

Figure 3 TTE apical view.

\section{Discussion}

Our little patient presented an ancommunicating hydrocephalus (acquired disease process, characterized by an impairment of the cerebrospinal fluid resorption mechanisms), caused by intraventricular hemorrhage (dreaded complication of prematurity). The condition was treated with VA shunt due to failure of the previous VP shunt. The current literature report the thrombotic obstruction of atrial catheter as one of the most dangerous complications, while there is a lack of reliable data about its incidence since few studies have focussed on this problem. ${ }^{8,9}$ The few researches on the argument would seem to orient towards the surgical treatment as a first-line intervention, lifesaving, even in case of infection of the atrial catheter. In fact only in one study it was reported a positive outcome in two patients treated with anticoagulant medical therapy. ${ }^{10}$ Recent researches have also linked the increased incidence of thrombotic events with genetic disorders of coagulation, ${ }^{11}$ and therefore suggest to comprehensively study the coagulation factors of the patients to reduce the recurrency of those events. ${ }^{12}$

Finally, an interesting rare case of obstruction of the SVC in an adult patient with VA shunt was solved without surgery, but by catheter-directed thrombolysis, followed by stenting of the SVC. This method would seem to hinder the reocclusion in cases of obstruction and relative stenosis of the SVC,but to this day, it hasn't been used in children. In the early childhood the only available treatment is constituted by the anticoagulant ${ }^{13,14}$ and antibiotic therapy; however, if there are associated signs of endocarditis, these therapies do not appear decisive, but are connected to a sudden surgical treatment. Our experience is in line with the data reported in the literature ${ }^{15,16}$ and it has clearly shown the inadequacy and limitations of the currently available medical therapy ${ }^{17,18}$ and the need for a timely surgical intervention strategy. This evidence should be correlated to the fact that the presence of the foreign body (the catheter) perpetuates an endothelial damage, a key element already known to be the first cause in the genesis of thrombotic phenomena, as described by Virchow in his famous triad. It should also be considered that aiming to the surgery, the only therapy that has shown partial efficacy has been the fibrinolytic one. However, the use of this pharmaceutical in the pediatric age carries many risks, even because there aren't standardized protocols. We chose as thrombolytic rTPA,${ }^{19}$ that although it possess a selective fibrin-specific action, it has also very famous side effect such as hemorrhagic events, hypersensitivity which can reach up to the shock, cardiac rhythm disturbances and severe episodes of low blood pressure. These side effects were observed more frequently in case of elongated or repeated treatments, that therefore we discourage.

\section{Conclusion}

From the management of this case,we learned that in similar condition the anticoagulant medical treatment, shall be deemed as "single and short-term" medical approach and it has to be followed in the shortest possible time by a resolutive surgical intervention.

\section{Acknowledgments}

None.

\section{Conflicts of interest}

Author declares there is no conflicts opf interest.

\section{Funding}

None.

\section{References}

1. Schmidt B, Andrew M. Neonatal thrombosis: report of a prospective Canadian and international registry. Pediatrics. 1995;96(5 Pt 1):939-943.

2. van Ommen CH, Heijboer H, Büller HR, et al. Venous thromboembolism in childhood: a prospective two-year registry in The Netherlands. $J$ Pediatr . 2001;139(5):676-681.

3. Nowak-Göttl U, von Kries R, Göbel U. Neonatal symptomatic thromboembolism in Germany: two year survey. Arch Dis Child Fetal Neonatal Ed. 1997;76(3):F163-F167.

4. Massicotte MP, Sofronas M, deVeber G. Difficulties in performing clinical trials of antithrombotic therapy in neonates and children. Thromb Res. 2006;118(1):153-163.

5. Veldman A, Nold MF, Michel-Behnke I. Thrombosis in the critically ill neonate: incidence, diagnosis, and management Vasc Health Risk Manag. 2008;4(6):1337-1348.

6. Monagle P, Chalmers E, Chan A, et al. Antithrombotic therapy in neonates and children: American College of Chest Physicians EvidenceBased Clinical Practice Guidelines (8th Edition). Chest. 2008;133(6 Suppl):887S-968S.

7. Roach ES, Golomb MR, Adams R, et al. Management of Stroke in Infants and Children: A Scientific Statement From a Special Writing Group of the American Heart Association Stroke Council and the Council on Cardiovascular Disease in the Young. Stroke. 2008;39(9):2644-2691. 
8. Thornburg C, Pipe S. Neonatal thromboembolic emergencies. Semin Fetal Neonatal Med. 2006;11(3):198-206.

9. Saxonhouse MA, Manco-Johnson MJ. The evaluation and management of neonatal coagulation disorders. Semin Perinatol. 2009;33(1):52-65.

10. Monagle P, Newall F, Barnes C, et al. Arterial thromboembolic disease: a single-centre case series study. $J$ Paediatr Child Health. 2008;44(1-2):28-32.

11. Saracco P, Parodi E, Fabris C, et al. Management and investigation of neonatal thromboembolic events: genetic and acquired risk factors. Thromb Res . 2009;123(6):805-809.

12. Williams MD, Chalmers EA, Gibson BE. The investigation and management of neonatal haemostasis and thrombosis. Br J Haematol . 2002;119(2):295-309.

13. Manco-Johnson MJ, Grabowski EF, Hellgreen M, et al. Recommendations for tPA thrombolysis in children. On behalf of the Scientific Subcommittee on Perinatal and Pediatric Thrombosis of the Scientific and Standardization Committee of the International Society of Thrombosis and Haemostasis. Thromb Haemost. 2002;88(1):157-158.
14. Paul M, Elizabeth Chalmers, Anthony Chan, et al. Antithrombotic Therapy in Neonates and Children: American College of Chest Physicians Evidence-Based Clinical Practice Guidelines (8th Edition). Chest. 2008;133(6_suppl):887S-968S.

15. Andrew M, Marzinotto V, Massicotte $P$, et al. Heparin therapy in pediatric patients: a prospective cohort study. Pediatr Res. 1994;35(1):78-83.

16. Newall F, Johnston L, Ignjatovic V, et al. Unfractionated heparin therapy in infants and children. Pediatrics. 2009;123(3):e510-e829.

17. Greenway A, Massicotte MP, Monagle P. Neonatal thrombosis and its treatment. Blood Rev. 2004;18(2):75-84.

18. Schmidt B, Andrew M. Neonatal thrombotic disease: prevention, diagnosis, and treatment. J Pediatr . 1988;113(2):407-410.

19. Wang M, Hays T, Balasa V, et al. Low-dose tissue plasminogen activator thrombolysis in children. JPediatr Hematol Oncol. 2003;25(5):379-386. 